\title{
$O\left({ }^{1} S\right)$ and $O\left({ }^{1} D\right)$ emission lines in the spectrum of 153P/2002 C1 (lkeya-Zhang)
}

\author{
M. T. Capria ${ }^{1}$, G. Cremonese ${ }^{2}$, A. Bhardwaj ${ }^{3, \star}$, and M. C. De Sanctis ${ }^{1}$
}

\author{
1 INAF - Istituto di Astrofisica Spaziale e Fisica Cosmica (IASF), Area di Ricerca di Tor Vergata, \\ via del Fosso del Cavaliere 100, 00133 Roma, Italy \\ e-mail: mariateresa.capria@rm.iasf.cnr.it \\ 2 INAF - Padua Observatory, vicolo dell'Osservatorio, Padua, Italy \\ 3 Space Physics Laboratory, Vikram Sarabhai Space Centre, Trivarundrum 695022, India
}

Received 13 March 2005 / Accepted 10 June 2005

\section{ABSTRACT}

High resolution spectroscopy observation of comet 153P/2002 C1 Ikeya-Zhang was performed on April 20, 2002 with the echelle spectrograph SARG on the $3.5 \mathrm{~m}$ Telescopio Nazionale Galileo in La Palma. The atomic O emission lines at 5577, 6360 and $6363 \AA$ are clearly visible in the spectrum. We determined the intensity ratio of the green line to the sum of the red lines. In order to verify the hypothesis that water is the main parent species of these emissions, the result was compared with the value obtained from a coupled chemistry-transport model. The measured value for the green to red ratio, $0.12 \pm 0.1$, agrees quite well with the value obtained from the model. This led us to conclude that during the observation water was the parent species of atomic $\mathrm{O}$ lines.

Key words. comets: individual: 153P/2002 C1 Ikeya-Zhang - comets: general

\section{Introduction}

It has been known since the early seventies (Blamont \& Festou 1974; Keller 1973a,b) that water ice is the main constituent of cometary nuclei, and as a consequence gaseous $\mathrm{H}_{2} \mathrm{O}$ and its dissociation products $(\mathrm{OH}, \mathrm{O}$ and $\mathrm{H})$ are the dominant species in the coma starting from a heliocentric distance of 3-4 AU. Unfortunately, the water molecule is difficult to detect, while it is much easier to detect its main dissociation product $\mathrm{OH}$ at radio and near UV wavelengths, and in this way it is possible to retrieve the properties of the parent: for this reason $\mathrm{OH}$ has been extensively studied.

For atomic oxygen, three important emission lines can be observed in the visible part of spectra with high enough spectral resolution: the green line at $5577.339 \AA\left({ }^{1} \mathrm{~S}-{ }^{1} \mathrm{D}\right)$ and the forbidden red oxygen doublet at 6300.304 and $6363.776 \AA$ $\left({ }^{1} \mathrm{D}-{ }^{3} \mathrm{P}\right)$. A fourth line at $2972.288 \AA$ has been seen only once (Festou \& Feldman 1981) but its detection has not been confirmed. Biermann \& Treffz (1964) were the first to suggest an excitation mechanism for these lines; later on it was discussed in detail by Festou \& Feldman (1981). These lines cannot be produced by solar resonance fluorescence excitation of the ground-state oxygen atom, but represent a "prompt emission", which means that they can be produced directly in the

* Currently at NASA Marshall Space Flight Center, NSSTC/XD12, Huntsville, Alabama 35805, USA. excited ${ }^{1} \mathrm{D}$ and ${ }^{1} \mathrm{~S}$ states by photodissociation of the parent molecule.

There are many reactions that can produce these forbidden oxygen lines (Bhardwaj \& Haider 2002). However, not all of them involve the water molecule: they can also involve $\mathrm{CO}$ and $\mathrm{CO}_{2}$. The question of the origin of $\mathrm{O}$ lines is interesting for two reasons. The first one is that, because the lifetime of the ${ }^{1} \mathrm{D}$ state is $\sim 110 \mathrm{~s}$, much shorter than the corresponding lifetime of water molecule $\left(\sim 8 \times 10^{4} \mathrm{~s}\right)$ at $1 \mathrm{AU}$, the red emission lines are a good tracer of water molecule distribution in the cometary coma. The second and more important reason is that, if water is the main producer of these lines, then from the O column density the $\mathrm{H}_{2} \mathrm{O}$ production rate can be deduced (see, for example, Morgenthaler et al. 2001, 2004).

One or more of these lines were detected many times (Fink \& Johnson 1984; Magee-Sauer et al. 1990; Schultz et al. 1993; Morrison et al. 1997; Cochran 1984; Cochran \& Cochran 2001; Zhang et al. 2001; Morgenthaler et al. 2001, reporting the more recent papers), but their unambiguous detection, especially the line at $5577 \AA$ is a non-trivial task. Firstly, high spectral resolution is necessary to discriminate the three lines from the usually strong telluric $\mathrm{O}$ emission lines. Secondly, the red line at $6300 \AA$ is very close to the $\mathrm{Q}$ branch of a $\mathrm{NH}_{2}$ band, and the green line at $5577 \AA$ is in the middle of a crowded $\mathrm{C}_{2}$ band $((1,2)$ P-branch). 
Table 1. Observing parameters.

\begin{tabular}{lc}
\hline \hline UT Date & 20 Apr. 2002 \\
$r(\mathrm{AU})$ & 0.89 \\
$\dot{r}\left(\mathrm{~km} \mathrm{~s}^{-1}\right)$ & 29.06 \\
$\Delta(\mathrm{AU})$ & 0.43 \\
$\dot{\Delta}\left(\mathrm{km} \mathrm{s}^{-1}\right)$ & -8.3 \\
Exposure time $(\mathrm{s})$ & 2400 \\
\hline
\end{tabular}

\section{Observations and reduction}

Discovered in 2002 by a Japanese and a Chinese amateur astronomers, the comet Ikeya-Zhang was initially designated C/2002 C1 (Ikeya-Zhang). Later on, Hasegawa \& Nakano (2002) identified it as the comet C/1661 C1 (Hevelius) and two historical comets appearing in 877 and 1273 . The comet turned out to be periodic (with an unusual, "intermediate" orbital period of $\sim 400$ years) and was then given the name 153P/IkeyaZhang.

We observed the comet on April 20, 2002 with the cross dispersed echelle spectrograph SARG on the $3.5 \mathrm{~m}$ Telescopio Nazionale Galileo in La Palma (Canary Islands, Spain) (Capria et al. 2002). During the observation the comet had a heliocentric distance of $0.89 \mathrm{AU}$ and a geocentric distance of $0.43 \mathrm{AU}$ (Table 1). We used a slit with $0.80 \times 5.3$ arcsec and the yellow grism covering a spectral range of 4620-7920 $\AA$ on the mosaic of two CCDs, providing a resolving power $R=57000$. The data along the slit length, corresponding to $1627 \mathrm{~km}$ on the comet, were summed, obtaining a one-dimensional spectrum.

The data were reduced using the IRAF ECHELLE package. Incandescent lamp observations were used for flat field correction, and the ThAr lamp for the wavelength calibration. By fitting the thorium line positions for all orders a dispersion solution was achieved with rms errors lower than $7 \mathrm{~mA}$. 55 orders (34 on the blue chip and 21 on the red chip) were obtained in the range $4620-7920 \AA$. The quality of data is very good and thousands of lines are visible. We are presently listing and identifying all these lines by comparison with existing catalogs (Cochran \& Cochran 2002; Brown et al. 1996; Zhang et al. 2001). The cometary atomic oxygen green line and the red doublet are clearly visible in the orders 24 of the blue part and 2 and 3 of the red part, respectively (see Figs. 1-3). In the orders selected to study the oxygen ratio, the continuum has been corrected in a different way with respect to the other orders. The continuum under these emission lines does not have particularly deep features, so it can be fitted using a cubic spline of second order obtaining a very good fit with $\mathrm{rms}<2 \%$; the result of the fit has then been subtracted from the order spectrum.

Looking at the plot of Fig. 4, which is an enlarged view of Fig. 1 showing the green line, the telluric line, while lying very close to the cometary line, is clearly distinguishable. The surrounding $\mathrm{C}_{2}$ lines are much weaker than the $\mathrm{O}$ lines; altough some contamination is probably present, it is not substantial. The cases of the red doublet lines are similar (Figs. 5 and 6): the telluric lines are blended with the cometary lines, but their contribution can still be well separated.

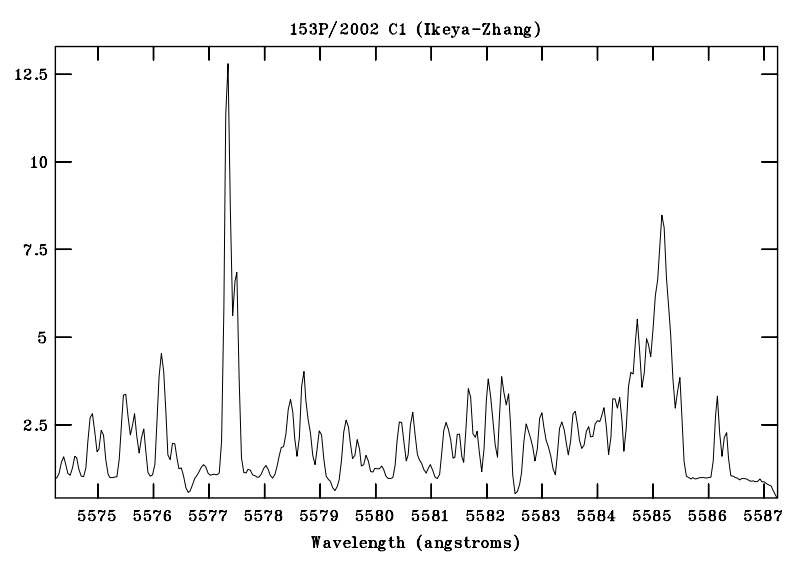

Fig. 1. Oxygen atomic line at $5577.339 \AA$ (green line). The units of the vertical axis are arbitrary units.

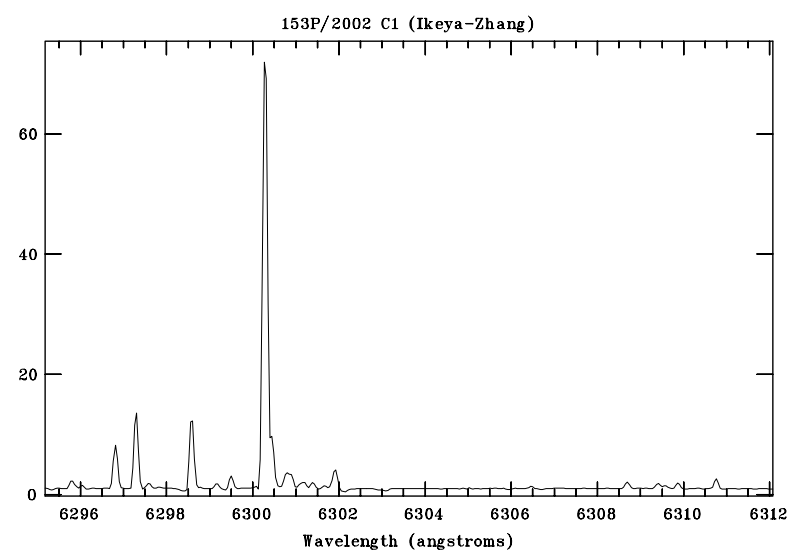

Fig. 2. Oxygen atomic line at $6300.304 \AA$ (red doublet). The units of the vertical axis are arbitrary units.

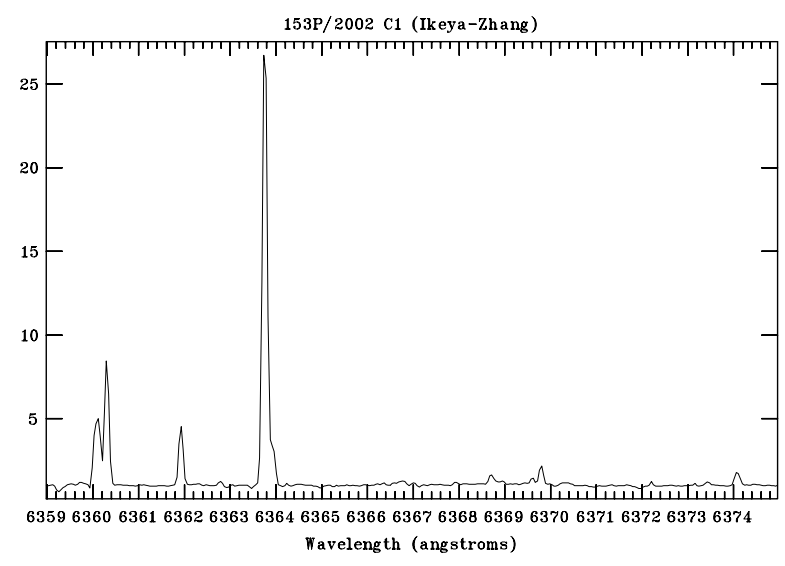

Fig. 3. Oxygen atomic line at $6363.776 \AA$ (red doublet). The units of the vertical axis are arbitrary units.

\section{The ratio of the atomic oxygen lines}

The ratio of the two red lines can give us a first idea of the quality of our data. The intensity of an emission line can be written as:

$I=10^{-6} \tau_{p}^{-1} \alpha \beta N$

where $\tau$ is the dissociative lifetime of the parent, $\alpha$ is the yield of photodissociation, $\beta$ is the branching ratio and $N$ is the 


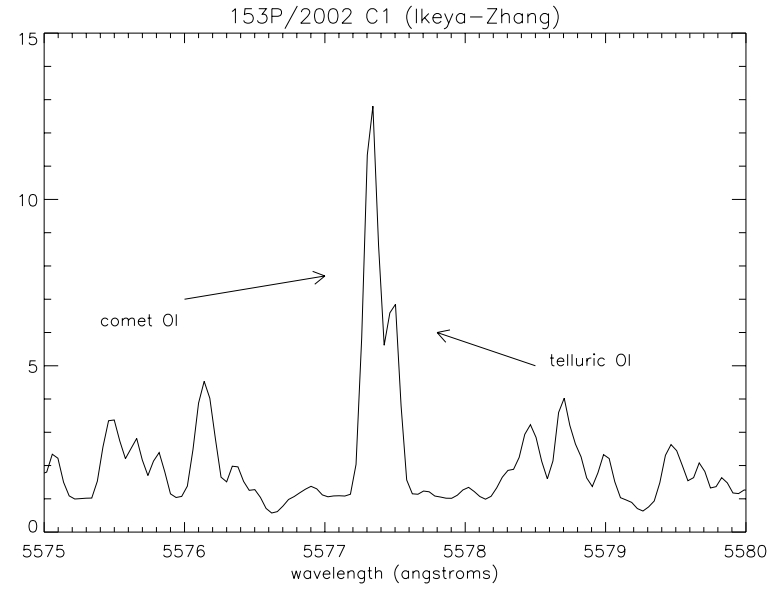

Fig. 4. Oxygen atomic line at $5577 \AA$ (enlarged view of Fig. 1). The telluric line is visible. The units of the vertical axis are arbitrary units.

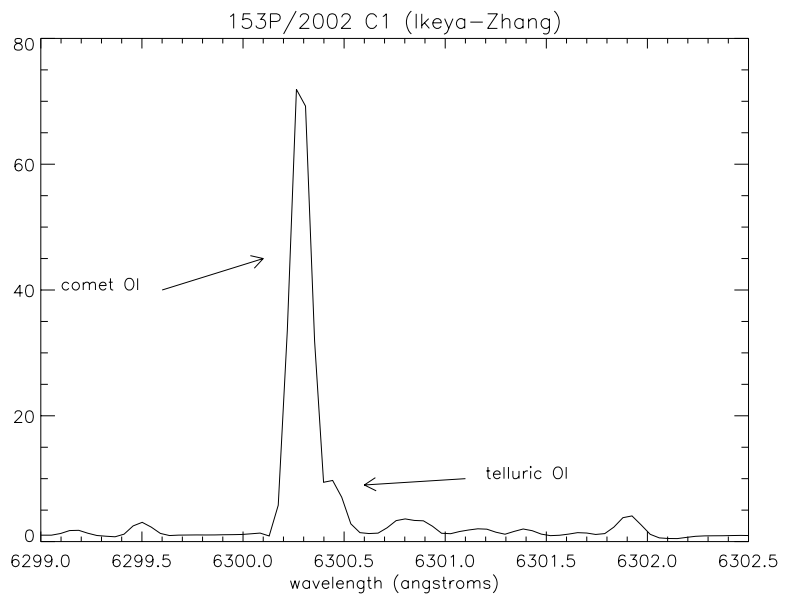

Fig. 5. Oxygen atomic line at $6363 \AA$ (enlarged view of Fig. 2). The telluric line is visible.The units of the vertical axis are arbitrary units.

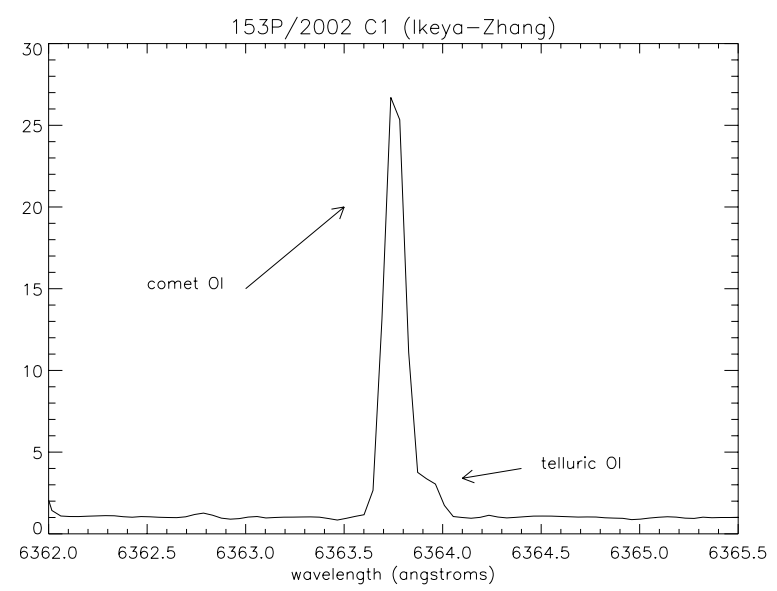

Fig. 6. Oxygen atomic line at $6363 \AA$ (enlarged view of Fig. 3). The telluric line is visible. The units of vertical axis are arbitrary units.

column density of the parent molecule (Cochran \& Cochran 2001). In the case of the red doublet, the ratio of the two line strengths should be the same as the ratio of the branching ratios, because they are both transitions from the $\left(2 \mathrm{p}^{4}\right)^{1} \mathrm{D}$ state to
Table 2. Theoretical value of the ratio $\mathrm{O}\left({ }^{1} \mathrm{~S}\right) / \mathrm{O}\left({ }^{1} \mathrm{D}\right)$ (Festou \& Feldman 1981).

\begin{tabular}{cc}
\hline \hline Parent & Ratio $\mathrm{O}\left({ }^{1} \mathrm{~S}\right) / \mathrm{O}\left({ }^{1} \mathrm{D}\right)$ \\
\hline $\mathrm{H}_{2} \mathrm{O}$ & $\sim 0.1$ \\
$\mathrm{CO}_{2}$ & $\sim 1$ \\
$\mathrm{CO}$ & $\sim 1$ \\
\hline
\end{tabular}

the $\left(2 \mathrm{p}^{4}\right)^{3} \mathrm{P}$ ground state. In our case, the value of the ratio is $2.9 \pm 0.1$, in good agreement with the value of 3.027 given by Storey \& Zeippen (2000).

The intensity ratio of the green to the sum of the two red lines is much more interesting because it allows to verify the assumption that the parent molecule is mainly water. In the case of the green to red intensity ratio we can write:

$$
\frac{I_{5577}}{I_{6300}+I_{6364}}=\frac{\tau_{\text {green }}^{-1} \alpha_{\text {green }} N_{\text {green }} \beta_{5577}}{\tau_{\text {redn }}^{-1} \alpha_{\text {red }} N_{\text {red }}\left(\beta_{6300}+\beta_{6364}\right)}
$$

and, if we assume that there is only one parent, the column densities are almost the same. For this ratio we obtain a value of $0.12 \pm 0.1$; the quoted error takes into account possible contributions from surrounding lines in the evaluation of the oxygen line intensities. The effective excitation ratio for dissociation of a parent molecule is proportional to $\tau^{-1} \alpha \beta$ : the value we obtained can be compared with the values listed in Table 2, taken from Festou \& Feldman (1981). Our value seems to be consistent with the production of $\mathrm{O}$ atoms predominantly from the $\mathrm{H}_{2} \mathrm{O}$ dissociation.

\section{Comparison with theoretical values}

The values for the two ratios described above can be compared with the values obtained from a coupled chemistry-transport model developed to study the chemistry of $\mathrm{O}$ atoms and their emissions. A brief description of this model will be given in the following subsection, while in the subsection 4.2 our derivation of the gas production rates of Ikeya-Zhang along the orbit will be described. The theoretical values of the ratios will be discussed in the Sect. 4.3 .

\subsection{Chemistry-transport model of $O$ atoms}

The coupled chemistry-transport model developed by Bhardwaj and co-workers (Bhardwaj et al. 1996; Bhardwaj \& Haider 1999; Bhardwaj 1999) has been employed to simulate the chemistry of $\mathrm{O}\left({ }^{1} \mathrm{D}\right)$ and $\mathrm{O}\left({ }^{1} \mathrm{~S}\right)$ atoms in the cometary coma. The degradation of the solar UV-EUV radiation and solar EUV-generated photoelectrons in the coma is modeled using the method of Bhardwaj et al. (1990, 1996) and further developed by Bhardwaj (2003). The electron impact production rates are calculated using the Analytical Yield Spectrum (AYS) approach, which is based on the Monte Carlo method. Details of AYS approach are given in several of the previous cited papers (Bhardwaj et al. 1990, 1996; Bhardwaj \& Michael 1999a,b; Bhardwaj 1999). The current model takes into account the most recently published cross sections for electron impact dissociation, ionization, and dissociative ionization 
processes for the gases in the coma. The basic chemical model for the $\mathrm{O}\left({ }^{1} \mathrm{D}\right)$ atoms used in this work is described in Bhardwaj \& Haider 2002. More details of the $\mathrm{O}\left({ }^{1} \mathrm{D}\right)-\mathrm{O}\left({ }^{1} \mathrm{~S}\right)$ model will be presented in a separate paper (Bhardwaj et al. 2005).

\subsection{Derivation of gas production rates}

The production rates along the orbit of Ikeya-Zhang for water, $\mathrm{CO}_{2}$ and $\mathrm{CO}$ have been derived through a nucleus evolution model. This nucleus model, developed by our group, was applied many times to real comets; hereafter a short description follows, for more details the reader is referred to Capria et al. (2000, 2001), Capria (2002), De Sanctis et al. (2001, 2003).

The uni-dimensional model considers a spherical nucleus, porous and initially homogeneous, composed of different ices and a refractory component, described as distributions of spherical grains.

A system of coupled equations, expressing energy and mass conservation, is solved for the whole nucleus:

$\rho c \frac{\partial T}{\partial t}=\nabla[k \cdot \nabla T]+\sum_{i=1}^{n} Q_{i}+Q_{\mathrm{am}-\mathrm{cr}}$

$\frac{1}{R T} \frac{\partial P_{i}}{\partial t}=\nabla\left[G_{i} \cdot \nabla P_{i}\right]+Q^{\prime} i \quad i=1, n$

where $Q_{i}$ are the energies exchanged by the solid matrix in the sublimation and recondensation of the ices, $Q_{\mathrm{am}-\mathrm{cr}}$ is the heat released during the transition of water ice from amorphous to crystalline form, $R$ is the gas constant, $P_{i}$ the partial pressure of component $i, G_{i}$ its diffusion coefficient, and $Q^{\prime}{ }_{i}$ is the gas source term due to sublimation-recondensation processes.

The temperature on the surface is obtained by a balance between the solar energy reaching the surface, the energy reemitted in the infrared, the heat conducted to the interior and the energy used to sublimate surface ices. Due to the rising temperature, ices start to sublimate, beginning with the more volatile species. The initial homogeneous nucleus differentiates giving rise to a layered structure in which the boundary between different layers is a sublimation front. When the ices near the surface of the nucleus begin to sublimate, the refractory particles become free and undergo the drag exerted by the escaping gas, so that they can be blown off or they can accumulate on the surface to form a crust.

Such a model depends on many parameters describing the orbit, the initial conditions of the nucleus and its physical properties. These parameters are taken as far as possible from observations; however, wherever no observational value is available, a value considered to be "typical" for most comets is used. In the present case, the dust/gas ratio was assumed to be 1 , the nucleus is made of refractory particles and ices of water, $\mathrm{CO}_{2}$ and $\mathrm{CO}$. The initial values of $\mathrm{CO}_{2}$ and $\mathrm{CO}$ with respect to water are 0.01 and 0.05 respectively. Figure 7 presents the gas fluxes obtained from this model; the vertical line marks the heliocentric distance of our observation. Only a part of the post-perihelion branch of the orbit is shown.

The theoretical curves can be compared with the values derived from observations. The comet Ikeya-Zhang was observed with the echelle spectrometer at the NASA Infrared

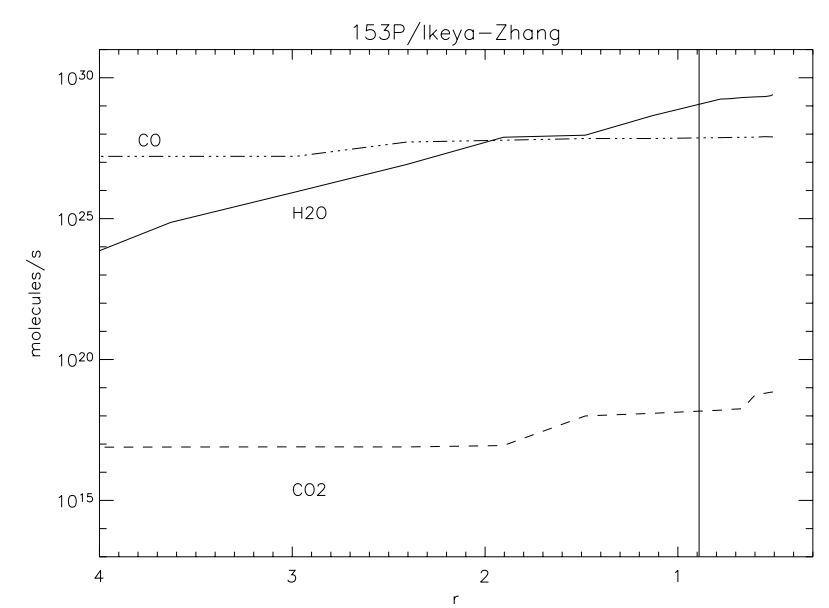

Fig. 7. $\mathrm{H}_{2} \mathrm{O}, \mathrm{CO}_{2}$ and $\mathrm{CO}$ fluxes along the post-perihelion branch of the orbit of Ikeya-Zhang from the nucleus model; the vertical bar marks the heliocentric distance at the time of observation.

Telescope Facility on Mauna Kea, Hawaii, between March 21 and April 13, 2002 (0.51 and 0.78 AU post-perihelion). From several $\mathrm{H}_{2} \mathrm{O}$ hot-band lines near $2.9 \mu \mathrm{m}$ a water production rate curve was deduced (Dello Russo et al. 2004): $Q_{\mathrm{H}_{2} \mathrm{O}}=$ $(9.2 \pm 1.1) \times 10^{28}\left[r^{(-3.21 \pm 0.26)}\right]$ molecules $\mathrm{s}^{-1}$. The analysis of the spatial profiles showed that most of water was released directly from the nucleus (Dello Russo et al. 2004). The mixing ratio of $\mathrm{CO}$ in the coma was $(4.7 \pm 0.8) \times 10^{-2}$ at $0.78 \mathrm{AU}$ postperihelion (Di Santi et al. 2002). Comet Ikeya-Zhang showed a moderately high gas-to-dust ratio from 2.7 AU to the perihelion according to Schleicher \& Birch (2002) observations. The agreement between the theoretical curves in Fig. 7 and the values derived from observations is good, considering the approximations made in the model and the error bars of the observed points.

\subsection{Derivation of the theoretical values for the $O$ lines ratios}

When the observation of comet Ikeya-Zhang was performed the projection of the slit, centered at the comet nucleus, corresponded to a length of $1627 \mathrm{~km}$. For the comet Ikeya-Zhang (assuming a water production rate of $2 \times 10^{29} \mathrm{~s}^{-1}$ ) this means that we are looking into the inner coma region. The major source of production of meta-stable O-species in the inner coma is the dissociation of $\mathrm{H}_{2} \mathrm{O}$, while the major loss processes are quenching (collision reactions with $\mathrm{H}_{2} \mathrm{O}$ ) and radiative deexcitation.

In the chemistry-transport model calculations, the total gas production rate for the comet Ikeya-Zhang is taken as $2 \times 10^{29}$ molecules $\mathrm{s}^{-1}$ on April 20, 2002 observation; the abundances of $\mathrm{CO}$ and $\mathrm{CO}_{2}$ are taken as $4.7 \%$ and $3 \%$ (relative to water). Other major O-bearing parent species (Bockelée-Morvan et al. 2004), e.g., $\mathrm{CH}_{3} \mathrm{OH}(\sim 2.5 \%)$ and $\mathrm{H}_{2} \mathrm{CO}(\sim 0.62 \%)$, were not considered because they do not have any significant effect on the chemistry of $\mathrm{O}\left({ }^{1} \mathrm{D}\right)$ and $\mathrm{O}\left({ }^{1} \mathrm{~S}\right)$ atoms (Bhardwaj \& Haider 2002).

Following the method of calculation of oxygen red (6300 and $6363 \AA)$ and green $(5577 \AA)$ line emissions 
Table 3. Oxygen lines ratio in the literature.

\begin{tabular}{lllll}
\hline \hline Comet & $\mathrm{O}\left({ }^{1} \mathrm{~S}\right) / \mathrm{O}\left({ }^{1} \mathrm{D}\right)$ & $r(\mathrm{AU})$ & $\Delta(\mathrm{AU})$ & Reference \\
\hline C/Iras-Araki-Alcock & $0.022-0.034$ & 1.02 & & Cochran (1984) \\
C/1996 B2 Hyakutake & $0.12-0.15$ & $1.083-1.002$ & $0.120-0.119$ & Morrison et al. (1997) \\
C/1995 O1 Hale-Bopp & $0.22-0.18$ & $0.920-0.991$ & $1.322-1.627$ & Zhang et al. (2001) \\
C/1999 S4 LINEAR & $0.06 \pm 0.01$ & $0.97-0.79$ & $1.21-0.45$ & Cochran \& Cochran (2001) \\
153P/Ikeya-Zhang & $0.12 \pm 0.1$ & 0.89 & 0.43 & This work \\
\hline
\end{tabular}

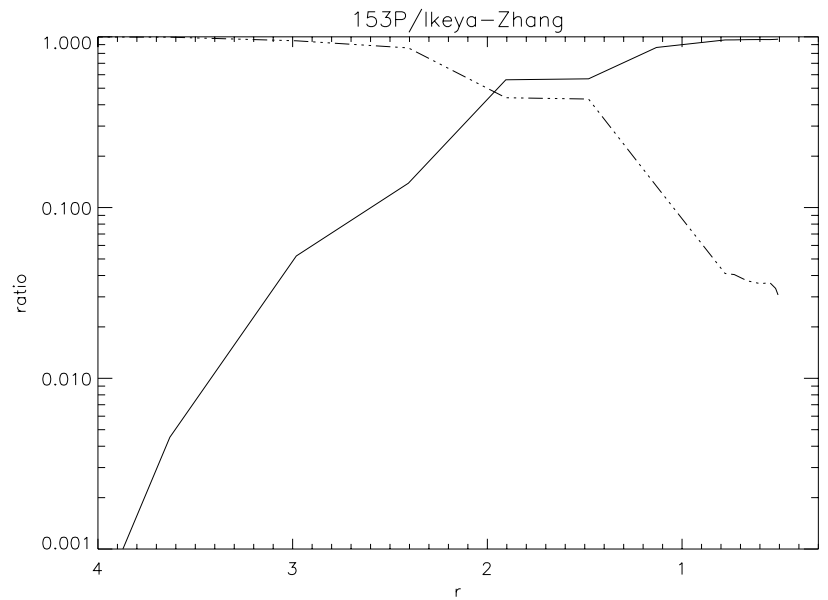

Fig. 8. Ratios of the fluxes of $\mathrm{H}_{2} \mathrm{O}$ (continuous line) and $\mathrm{CO}$ (dashdotted line) on the total gas flux from the nucleus model along the post-perihelion branch of the orbit of comet Ikeya-Zhang. The ratio of $\mathrm{CO}_{2}$ on the total flux is not visible at this scale.

(Bhardwaj \& Haider 2002; Bhardwaj et al. 2005), we derived the ratio of the intensities of the green to red lines. Our model calculated value is $0.1 \pm 0.01$. This value is in good agreement with the observed ratio value of $0.12 \pm 0.1$ stated in the preceding section.

\section{Discussion and conclusion}

Our observation is not the first measurement of the intensity ratio between the green and the red doublet lines of atomic oxygen in the comets. Table 3 presents this ratio for other comets, along with the heliocentric and geocentric distance of the comet at the observation time and the corresponding bibliographic reference.

From Table 3, it can be seen that all the measurements obtained point to the water as the only, or at least the dominant, parent of $\mathrm{O}$ atomic lines. However, the set of measured ratios is not representative, because all the ratios refer to comets at a heliocentric distance close to $1 \mathrm{AU}$, where it is well known that water ice sublimation is the main driver of the activity and the sublimated water constitutes the major part of the cometary gas. The behavior of gas production rates shown in Fig. 8 (where the variation of the ratios of $\mathrm{H}_{2} \mathrm{O}$ and $\mathrm{CO}$ fluxes from the nucleus model with respect to the total gas production is plotted along a part of the post-perihelion branch of the the cometary orbit), is very typical and can be considered as representative of the behavior of most of the short period comets. At the scale of the plot in Fig. 8 the $\mathrm{CO}_{2}$ ratio is not visible.
It would be very interesting to measure the ratio of green to red atomic $\mathrm{O}$ lines at a point along the cometary orbit where the sublimated water (and its products) is not the major constituent of the gaseous coma. We believe that at such times this ratio can be different from those shown in Table 3. We see from Table 3 that the highest ratio $(\simeq 0.2)$ was obtained for the comet HaleBopp. A possible reason is that the $\mathrm{CO}$ abundance with respect to water in this comet was very high $(\simeq 12 \%$ at the nucleus, and $\simeq 23 \%$ in the coma-extended source effect) (Bockelée-Morvan et al. 2004). As can be noted from Table 2, the ratio of green to red lines will tend towards unity with increasing abundance of $\mathrm{CO}$ and $\mathrm{CO}_{2}$ relative to water.

To conclude, the comparison of the model calculated and observed intensity ratio of the green to red lines of atomic oxygen lead us to say that $\mathrm{H}_{2} \mathrm{O}$ is the main parent species of the O lines on comet Ikeya-Zhang. However, in our opinion this conclusion holds only at heliocentric distances of $\sim 1 \mathrm{AU}$ from the Sun.

Acknowledgements. Based on observations made with the Italian Telescopio Nazionale Galileo (TNG) operated on the island of La Palma by the Fundación Galileo Galilei of the INAF (Istituto Nazionale di Astrofisica) at the Spanish Observatorio del Roque de los Muchachos of the Instituto de Astrofisica de Canarias.

The authors wish to thank C. Arpigny, whose comments were very helpful in improving the manuscript.

\section{References}

Bhardwaj, A. 1999, J. Geophys. Res., 104, 1929

Bhardwaj, A. 2003, Geophys. Res. Lett., 30, 2244

Bhardwaj, A., \& Haider, S. A. 1999, Adv. Space Res., 23, 1325

Bhardwaj, A., \& Haider, S. A. 2002, Adv. Space Res., 29, 745

Bhardwaj, A., \& Michael, M. 1999a, Geophys. Res. Lett., 26, 393

Bhardwaj, A., \& Michael, M. 1999b, J. Geophys. Res., 104, 24713

Bhardwaj, A., Haider, S. A., \& Singhal, R. P. 1990, Icarus, 85, 216

Bhardwaj, A., Haider, S. A., \& Singhal, S. P. 1996, Icarus, 120, 412

Bhardwaj, A., et al. 2005, in preparation

Biermann, L., \& Treffz, E. 1964, ZAp, 59, 1

Blamont, J. E., \& Festou, M. 1974, Icarus, 23, 538

Bockelée-Morvan, D., Crovisier, J., Mumma, M. J., \& Weaver, H. A. 2004, The composition of cometary volatiles, in Comets II, ed. M. C. Festou, U. Keller, \& H. A. Weaver (Tucson: Univ. Arizona Press), in press

Brown, M. E., Bouchez, A. H., Spinrad, H., et al. 1996, AJ, 112, 1197

Capria, M. T., Cremonese, G., Boattini, A., et al. 2002, High resolution spectroscopy of comet C/2002 C1 Ikeya-Zhang with SARG at TNG, in The Proceedings for the Asteroid, Comets, Meteors Conference, ed. B. Warmbein, ESA SP, 500, 693 
Capria, M. T. 2002, Earth, Moon and Planets, 89, 161

Capria, M. T., Coradini, A., De Sanctis, M. C., \& Orosei, R. 2000, A\&A, 357, 359

Capria, M. T., Coradini, A., De Sanctis, M. C., Blecka, M. I. 2001, Planetary and Space Sciences, 49, 907

Cochran, A. L., \& Cochran, W. D. 2001, Icarus, 154, 381

Cochran, A. L., \& Cochran, W. D. 2002, Icarus, 157, 297

Cochran, W. D. 1984, Icarus, 58, 440

Debi Prasad, C., Chandrasekhar, T., Desai, J. N., et al. 1988, PASP, 100,702

Dello Russo, N., Di Santi, M. A., Magee-Sauer, K., et al. 2004, Icarus, 168,186

De Sanctis, M. C., Capria, M. T., \& Coradini, A. 2001, AJ, 121, 2792

De Sanctis, M. C., Capria, M. T., \& Coradini, A. 2003, Adv. Space Res., 31, 2519

Di Santi, M. A., Dello Russo, N., Magee-Sauer, K., et al. 2002, Co, $\mathrm{H}_{2} \mathrm{CO}$ and $\mathrm{CH}_{3} \mathrm{OH}$ in Comet $\mathrm{C} / 2002 \mathrm{C} 1$ Ikeya-Zhang, in The Proceedings for the Asteroid, Comets, Meteors Conference, ed. B. Warmbein, ESA SP, 500, 571

Feldman, P. D. 1983, Science, 219, 347
Feldman, P. D., Festou, M. C., Tozzi, G. P., \& Weaver, H. A. 1997, ApJ, 475, 829

Festou, M. C., \& Feldman, P. D. 1981, A\&A, 103, 154

Fink, U., \& Johnson, J. R. 1984, AJ, 89, 1565

Keller, H. U. 1973, A\&A, 23, 269

Keller, H. U. 1973, A\&A, 27, 51

Hasegawa, I., \& Nakano, S. 2003, MNRAS, 345, 883

Magee-Sauer, K., Roesler, F. L., Scherb, F., \& Harlander, J. 1990, Icarus, 84,154

Morgenthaler, J. P., Harris, W. M., Scherb, F., et al. 2001, ApJ, 563, 451

Morgenthaler, J. P., Harris, W. M., Scherb, F., et al. 2004, BAAS, 36, 1145

Morrison, N. D., Knauth, D. C., Mulliss, C. L., \& Lee, W. 1997, PASP, 109,676

Schleicher, D. G., \& Birch, P. V. 2002, BAAS, 34, 853

Schultz, D., Li, G. S. H., Scherb, F., \& Roesler, F. L. 1993, Icarus, 101,95

Storey, P. J., \& Zeippen, C. J. 2000, MNRAS, 312, 813

Zhang, H. W., Zhao, G., \& Hu, J. Y. 2001, A\&A, 367, 1049 